\title{
Experimental Measurement of RCS Jet Interaction Effects on a Capsule Entry Vehicle
}

\author{
Gregory M. Buck, ${ }^{*}$ A. Neal Watkins, ${ }^{\dagger}$ Paul M. Danehy, ${ }^{\dagger}$ Jennifer A. Inman, ${ }^{\S}$ David W. Alderfer ${ }^{* *}$ \\ Artem A. Dyakonov ${ }^{\dagger \dagger}$ \\ NASA Langley Research Center, Hampton, VA, 23681
}

\begin{abstract}
An investigation was made in NASA Langley Research Center's 31-Inch Mach 10 Tunnel to determine the effects of reaction-control system (RCS) jet interactions on the aft-body of a capsule entry vehicle. The test focused on demonstrating and improving advanced measurement techniques that would aid in the rapid measurement and visualization of jet interaction effects for the Orion Crew Exploration Vehicle while providing data useful for developing engineering models or validation of computational tools used to assess actual flight environments. Measurements included global surface imaging with pressure and temperature sensitive paints and three-dimensional flow visualization with a scanning planar laser induced fluorescence technique. The wind tunnel model was fabricated with interchangeable parts for two different aft-body configurations. The first, an Apollo-like configuration, was used to focus primarily on the forward facing roll and yaw jet interactions which are known to have significant aft-body heating augmentation. The second, an early Orion Crew Module configuration (4-cluster jets), was tested blowing only out of the most windward yaw jet, which was expected to have the maximum heating augmentation for that configuration. Jet chamber pressures and tunnel flow conditions were chosen to approximate early Apollo wind tunnel test conditions. Maximum heating augmentation values measured for the Apollo-like configuration ( $>10$ for forward facing roll jet and 4 for yaw jet) using temperature sensitive paint were shown to be similar to earlier experimental results (Jones and Hunt, 1965) using a phase change paint technique, but were acquired with much higher surface resolution. Heating results for the windward yaw jet on the Orion configuration had similar augmentation levels, but affected much less surface area. Numerical modeling for the Apollo-like yaw jet configuration with laminar flow and uniform jet outflow conditions showed similar heating patterns, qualitatively, but also showed significant variation with jet exit divergence angle, with as much as 25 percent variation in heat flux intensity for a 10 degree divergence angle versus parallel outflow. These results along with the fabrication methods and advanced measurement techniques developed will be used in the next phase of testing and evaluation for the updated Orion RCS configuration.
\end{abstract}

\section{Nomenclature}

$A / R=$ jet exit to throat diameter area ratio

$c \quad=$ substrate specific heat

$C_{h} \quad=$ convective heat transfer coefficient based on enthalpy

$C_{h F R}=$ stagnation point convective heat transfer coefficient calculated using the method of Fay and Riddell

$C_{h A} / C_{h B}=$ heating augmentation, (ratio of surface heating with/without jet interaction)

$D_{1} \quad=$ capsule diameter $($ see Fig. 3$)$

\footnotetext{
* Aerospace Engineer, Aerothermodynamics Branch, MS 408A, AIAA Member.

${ }^{\dagger}$ Electronics Engineer, Advanced Sensing and Optical Measurement Branch, MS 493.

${ }^{\ddagger}$ Research Scientist, Advanced Sensing and Optical Measurement Branch, MS 493, AIAA Assoc. Fellow.

${ }^{\S}$ Research Scientist, Advanced Sensing and Optical Measurement Branch, MS 493, AIAA Member.

${ }^{* *}$ Research Scientist, Advanced Sensing and Optical Measurement Branch, MS 493.

${ }^{\dagger}$ Aerospace Engineer, Atmospheric Flight \& Entry Systems Branch, MS 489.
} 


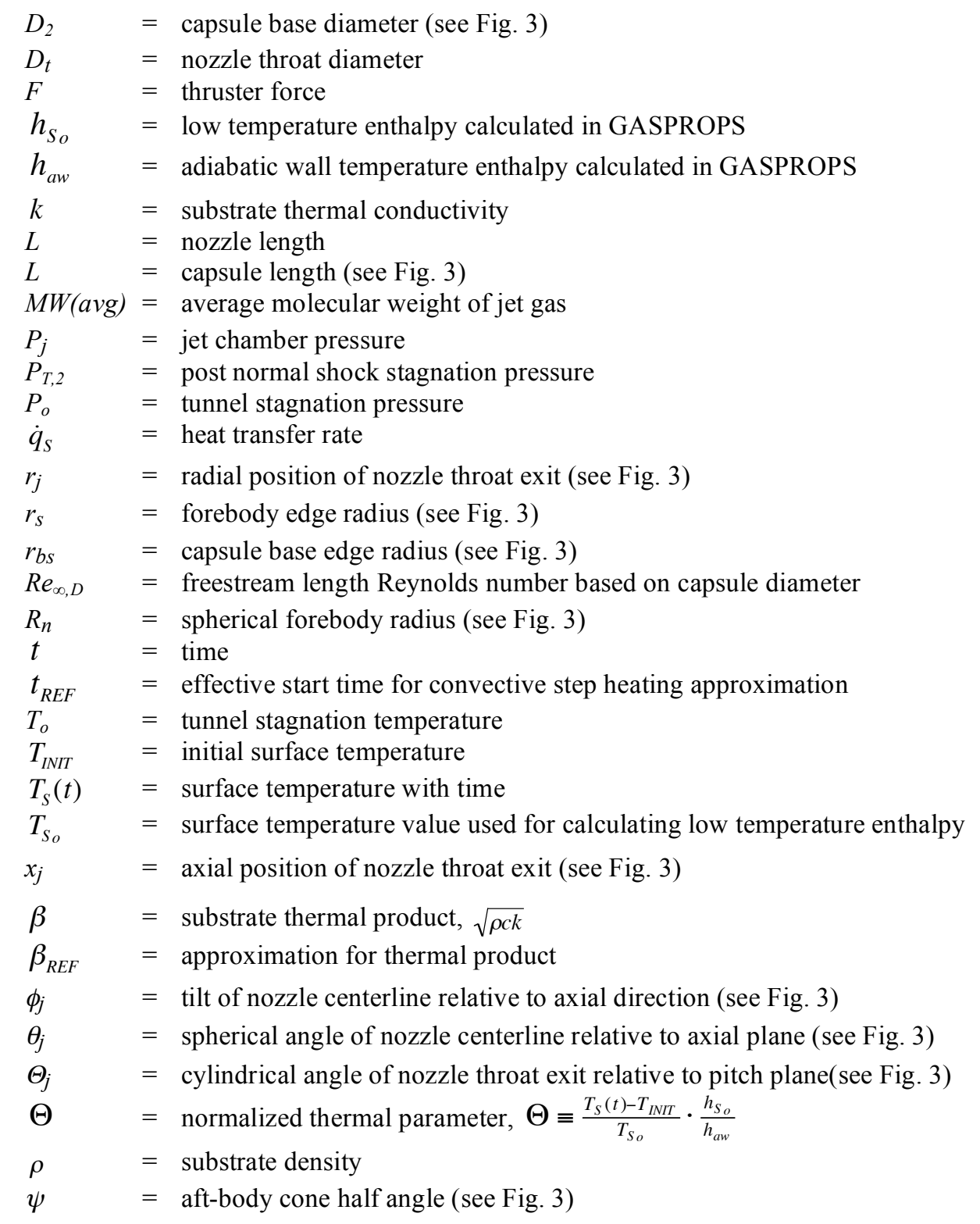

\section{Introduction}

dvanced experimental capabilities, under various stages of development, are being brought together at NASA A Langley Research Center to investigate the problem of reaction-control system (RCS) jet interactions affecting aft-body heating on the Orion Crew Module (OCM). To evaluate complex flow features and provide high resolution surface and flowfield measurements, techniques for complex model fabrication with internal plumbing and interchangeable nozzle configurations, optical surface pressure and temperature measurements and volumetric laser flowfield diagnostics are being developed and applied to the experimental problem. Plumes from the OCM RCS jets will alter the flowfield around and interact with the aft-body of the vehicle. The generic problem involves blunt body wake flow with cross-flow separation ahead of a jet plume (or multiple jet plumes) and highly threedimensional flowfield-surface interactions. Quoting from the summary of their AGARD report on the aerodynamic interference induced by reaction controls, Spaid and Cassel, 1973: "The jet interaction flowfield is so complex that predictions of induced forces, pressure distributions, concentration distributions, etc., must rely heavily on experimental data. Accurate predictions concerning situations which lie far outside the range of available data will require additional experimentation." In their particular case, they were studying centerline, or two-dimensional interactions on flat plates or bodies of rotation (missiles), all of which had fully developed boundary layers and 
attached flow. In the case of the OCM, it would be safe to say that RCS jet interaction effects lie outside the range of data that Spaid and Cassel were acquiring in the early 1970's and would require a unique experimental database to develop engineering models such as those used then or to validate computational tools used today. It is in preparation for generating such a database that the current experimental tools are being brought together and further developed. Models are being built using a stereolithography (SLA) rapid prototyping system for direct fabrication of internal passageways and interchangeable configurations. Surface pressure and heating effects are being measured using pressure and temperature sensitive paints (PSP and TSP) and corresponding flowfields around jet plumes are being visualized with a three-dimensional scanning planar laser induced fluorescence technique (PLIF).

\title{
II. Background
}

An early Apollo-era investigation by Jones and Hunt (1965) had similar objectives to the current study and had shown experimentally that the Apollo Command Module (ACM) forward facing roll and yaw RCS jets had significant effects on aft-body heating augmentation, with factors of 11 and 4 respectively. Heat transfer coefficients were obtained in that study in a Mach 8 tunnel using a new phase-change paint technique, which was recently developed at Langley Research Center (Jones and Hunt, 1964). Example heating patterns from the Apollo study by Jones and Hunt are shown in Figure 1. This original Apollo RCS jet interaction heating study was run on a model with a 33-degree aft-body cone angle and was tested at 33 degrees angle of attack. Shown are simple sublimated patterns with and without RCS jet interactions. Images were obtained from black and white photocopies of the original phase-change paint photographs. The left image shows a sublimated pattern from a run with jets off (for reference). The middle image shows a pattern with the forward roll jet on and the right image with yaw jet interactions. Edges of sublimated patterns indicate an isotherm, the temperature for which is unique to a given

(2-11 times heating augmentation reported)
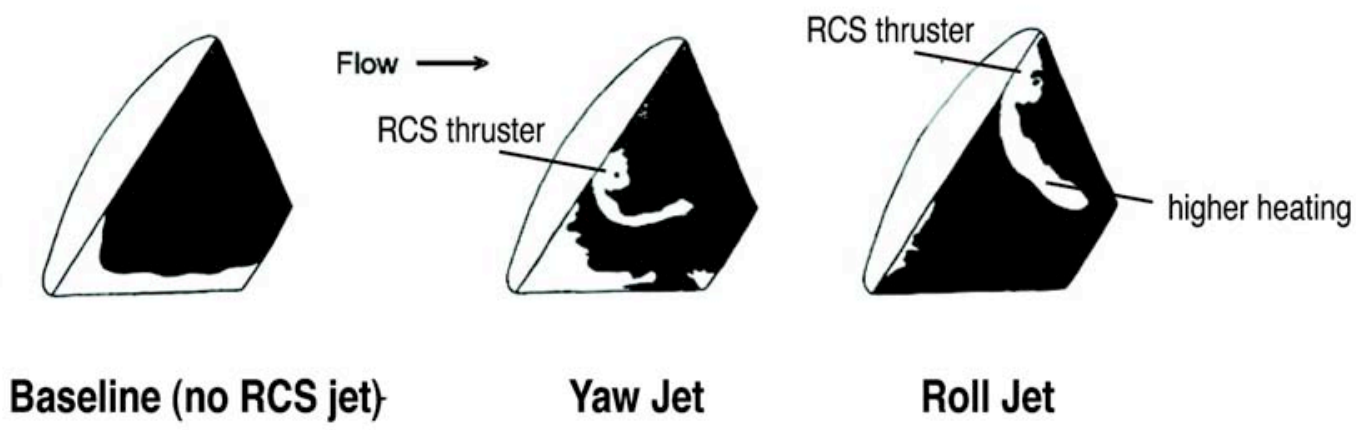

Yaw Jet

Roll Jet

\author{
Figure 1. Phase-Change Paint Heating Patterns on Apollo Command Module Aft-Body in \\ Langley Mach 8 Variable Density Tunnel (Jones and Hunt, 1965)
}

phase-change coating formulation. Surface heating coefficients are reduced from a series of timed photographs acquired during a run. A summary of the interpreted Apollo heating results is given in Table 1, with maximum heating augmentation values for each case given in the far right column.

An objective in the current test is to also measure surface heating for RCS jet configurations similar to Apollo with advanced techniques (TSP) and see how they compare. This would also provide confidence in testing a preliminary Orion configuration (4-cluster RCS jets) in which interaction heating effects are as yet unknown.

\section{Test Model Description}

An interchangeable capsule model was constructed with a fixed fore-body heat shield, internal high-pressure manifold and support sting fabricated from steel. The removable aft-body and RCS nozzle blocks were fabricated from a thermal insulating plastic/ceramic composite using a stereolithography apparatus (SLA) rapid prototyping system (Buck, 2006). The thermal insulating properties of this material make it ideal for aero-heating 
Table 1. List of Test Conditions and Maximum Heating Augmentation Values for RCS Jet Interference Heating in Jones and Hunt Study (1965)

\begin{tabular}{cccccc}
\hline \hline RCS Jet & $\boldsymbol{D}_{\boldsymbol{t}}$ (in.) & $\boldsymbol{R e}_{\infty, \boldsymbol{D}} \boldsymbol{x} \boldsymbol{1 0} \mathbf{0}^{\boldsymbol{6}}$ & $\boldsymbol{P}_{\boldsymbol{j}} / \boldsymbol{P}_{\boldsymbol{T}, \boldsymbol{2}}$ & $\boldsymbol{P}_{\boldsymbol{j}}, \mathbf{( p s i )}$ & $\boldsymbol{C}_{\boldsymbol{h} \boldsymbol{A}} / \boldsymbol{C}_{\boldsymbol{h} \boldsymbol{B}}$ \\
\hline yaw & 0.08 & 1.5 & 25 & 215 & 4 \\
yaw & 0.08 & 0.13 & 259 & 215 & 2.5 \\
yaw & 0.02 & 0.13 & 621 & 515 & 2 \\
yaw & 0.02 & 1.5 & 59 & 515 & 4 \\
pitch & 0.02 & 1.5 & 58 & 515 & 1 \\
yaw & 0.02 & 1.5 & 58 & 515 & 4 \\
forward roll & 0.02 & 1.5 & 59 & 515 & 4 \\
forward roll & 0.02 & 0.13 & 606 & 515 & 11 \\
aft roll & 0.02 & 0.13 & 606 & 515 & 3 \\
aft roll & 0.02 & 1.5 & 58 & 515 & 3 \\
\hline \hline
\end{tabular}

measurements. With the SLA rapid prototyping capability the number of RCS jets, nozzle shapes, locations and piping (flow on or off) can be rapidly and inexpensively re-configured.

Each aft-body fabricated had a 32.5-degree cone angle and 24-degree pitch angle relative to the support sting. These dimensions were chosen to be closer to the outer mold lines and attitude expected for the OCM. Aft-view comparisons of the model configurations are shown in Figure 2. On the left, OCM-A24, is the Orion 4-cluster RCS jet configuration with detailed cavities for the view ports, vents, umbilicals, launch abort system attachment wells and the rear pitch jets (not activated). The smooth body OCM model, OCM-A24, with only RCS jets and the smooth Apollo-like model, ACM-S24, with RCS jets configured similar to the model tested by Jones and Hunt are shown in the middle and right of Fig. 2. Each configuration is shown with pitch jets on top, yaw jets on the left centered $90^{\circ}$ from the top and roll jets situated between the pitch and yaw jets on either side. Dimensions and coordinates for each of the jets tested are given in Table 2 and Figure 3. Individual jets, tested and presented in the subsequent results section, include the forward facing roll and yaw jets from the ACM-S24 configuration and the windward yaw jet from the OCM-A24 configuration (most windward out of the four-jet yaw cluster).

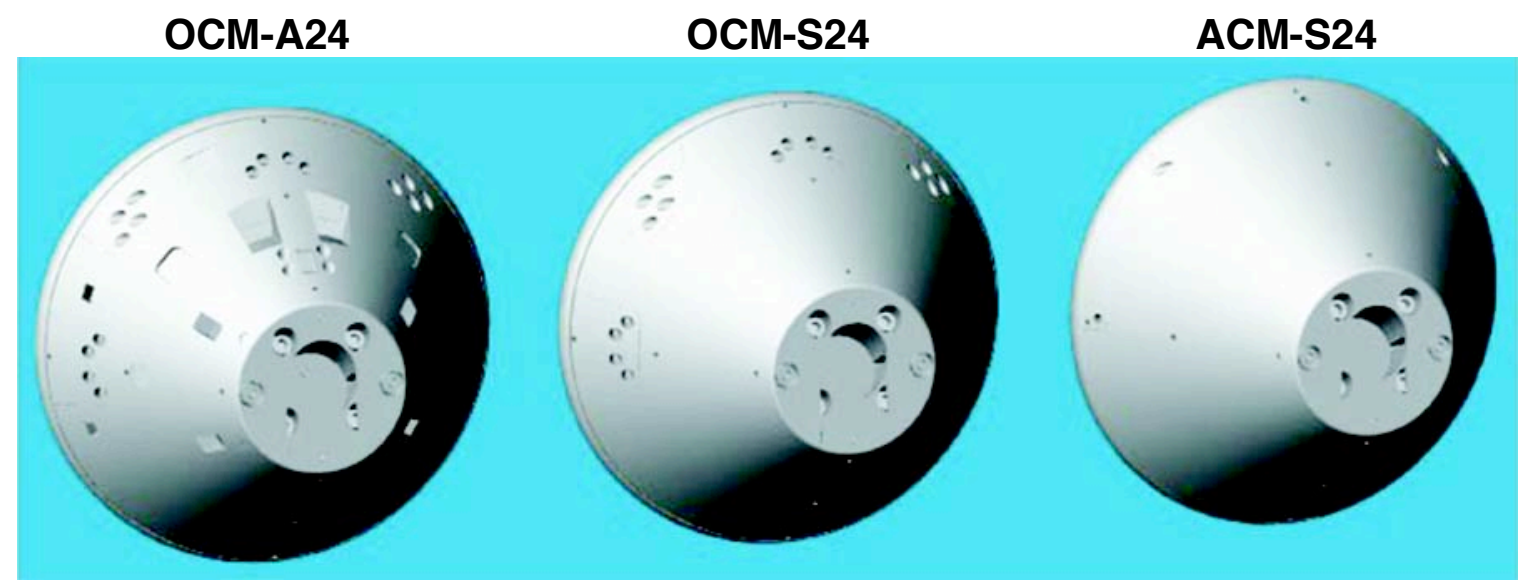

Figure 2. RCS Test Models - Aft View Comparisons

\section{Model Geometry}

All of the models were 5 -inch diameter, $2.53 \%$ full scale, with a 32.5 -degree aft-body half angle. Each had a base mounted cylindrical 0.5 -inch diameter sting that was orientated 24 degrees to the model axis of rotation and a sting-to-base diameter ratio of 0.28 . Nomenclature for the model dimensions and nozzle coordinates is shown in Figure 3. Values for the outer mold line dimensions and for each of the RCS jets tested are given in Table 2. Nozzle coordinates are listed by the position of the throat center in body cylindrical coordinates $(\mathrm{x}, \Theta, \mathrm{r})$ relative to the center of the heat shield surface and orientation of the nozzle centerline in local spherical angles $(\theta \mathrm{j}, \varphi \mathrm{j})$ relative to its radial position in the axial plane and tilt in the axial direction. 
Table 2. Outer Mold Line Dimensions and Nozzle Coordinates (See Figure 3 for Nomenclature)

\begin{tabular}{|c|c|c|c|c|c|c|c|}
\hline Model Designation & $D_{1}$ (in.) & $D_{2}$ (in.) & $R_{n}$ (in.) & $r_{s}$ (in.) & $r_{b s}$ (in.) & $L$ (in.) & $\psi(\mathrm{deg})$ \\
\hline ACM-S24 & 5 & 1.806 & 6 & 0.25 & 0.25 & 3.288 & 32.5 \\
\hline OCM-S24 & $"$ & $"$ & $"$ & " & " & " & " \\
\hline OCM-A24 & $"$ & $"$ & $"$ & $"$ & $"$ & $"$ & $"$ \\
\hline \multicolumn{2}{|c|}{ Nozzle Designation } & $x_{j}$ (in.) & $\Theta_{j}$ (deg) & $r_{j}$ (in.) & $\theta_{j}(\mathrm{deg})$ & $\phi_{j}$ (deg) & \\
\hline \multicolumn{2}{|c|}{ ACM yaw } & 0.897 & 88.5 & 2.182 & 0 & 50 & \\
\hline \multicolumn{2}{|c|}{ ACM forward roll } & 1.091 & 37.93 & 4.816 & 62.84 & 0 & \\
\hline \multicolumn{2}{|c|}{ ACM pitch* } & 0.897 & -1.5 & 2.182 & 0 & 50 & \\
\hline \multicolumn{2}{|c|}{ OCM windward yaw } & 1.345 & 98.608 & 1.951 & -8.608 & 74.818 & \\
\hline
\end{tabular}

* shown in PLIF flowfield images only

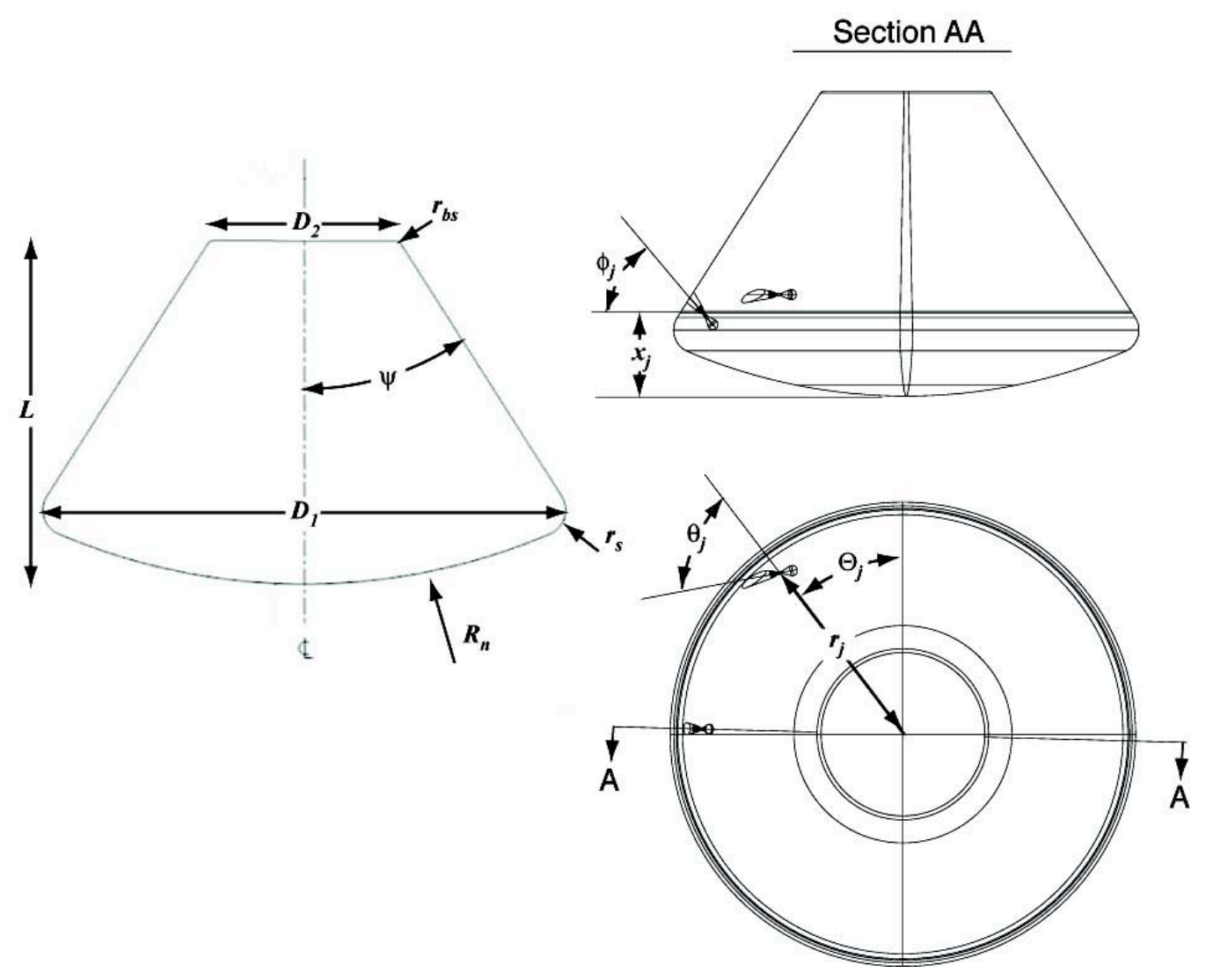

Figure 3. Outer Mold Lines and nozzle coordinate system for RCS Test Model.

Nozzle Geometry

Two different 10-degree nozzles were tested, each with 0.0275 -inch throat diameters. One is a simple cone with an exit to throat area ratio (A/R) of 13.4 and the other a bell/cone combination referred to simply as the "Bell" with $\mathrm{A} / \mathrm{R}=22.5$. Shapes of the two nozzles tested are shown in the bottom of Figure 4. The Bell nozzle was used in all of the test runs except for a series of PLIF runs in which the 10-degree Cone and Bell nozzles were compared in the pitch thruster location (providing a two-dimensional plane of symmetry for convenient single slice planar flow visualization).

Full-scale nozzle configurations are also shown in the top of Figure 4 for the 100 pound-force and 160 poundforce thrusters being considered for the flight vehicle. These comparisons are shown to highlight the differences in the model scale nozzles relative to full scale. In comparison with full scale, model-scale test nozzles have much 
Full Scale

L(in), $D_{1}$ (in), $F\left(l b_{4}\right.$ ), $P_{1}$ (psi), MW(avg)
Model Scale

$L$ (in), $D_{t}$ (in), $F\left(l_{f}\right.$ ), $P_{1}$ (psi), MW

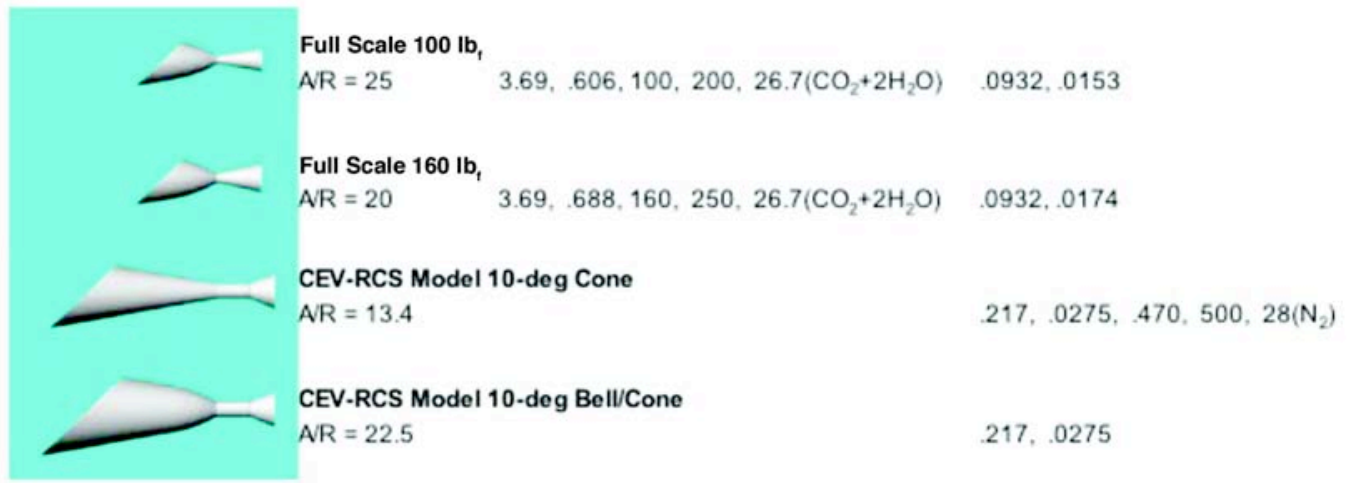

Figure 4. Comparison of RCS Nozzle Configurations .

larger throat diameters $\left(\mathrm{D}_{\mathrm{t}}\right)$ and throat lengths $(\mathrm{L})$ relative to scaled nozzle size. This is necessary to machine throat dimensions within a repeatable accuracy and test with reasonable chamber pressures. Unfortunately, nozzle exit areas also tend to be larger relative to scaled model features when throat diameters are enlarged. This concern is particularly compounded if clusters of jets are being tested, as is the case with the Orion configuration, particularly if multiple jets are being fired at once. The impact of jet exit area relative to model scale and multiple jet firing might need to be addressed in further studies.

Genesis for the current model nozzle designs descended from work on recent Mars Science Lander (MSL) RCS jet experiments using experience gained from Shuttle Orbiter RCS interaction tests (Scallion, 1995). The current 10-degree Cone nozzle is identical to the 10-degree MSL nozzle used by Scallion. For MSL this nozzle performed well at chamber pressures up to 500 psi in the Mach 10 tunnel and has been used in supersonic facilities with up to 1500 psi jet chamber pressures. In contrast, earlier tests (Danehy, 2006), using a 14-degree cone nozzle, had large jet exit divergence angles acting almost as a point source at chamber pressures above 80 psi. Model-scale nozzle performance and resulting plume shapes in the wind tunnel are important factors to consider in designing wind-tunnel experiments for RCS testing.

\section{Model Materials and Assembly}

An exploded view of the RCS test model is shown in Figure 5. The fore-body heat shield, internal highpressure manifold and support sting are fabricated from steel. Aft-body and RCS nozzle blocks are fabricated from a plastic/ceramic composite SLA material which works well for aero-heating substrates since it is also a thermal insulating material.

SLA parts are coated directly with a TSP for global surface heating measurements. Interchangeable SLA parts are also manufactured for pressure measurements using PSP, but are first plated with copper $(0.025$ " thick) to dissipate heat and provide a uniform temperature surface. Reducing temperature gradients significantly improves PSP measurement

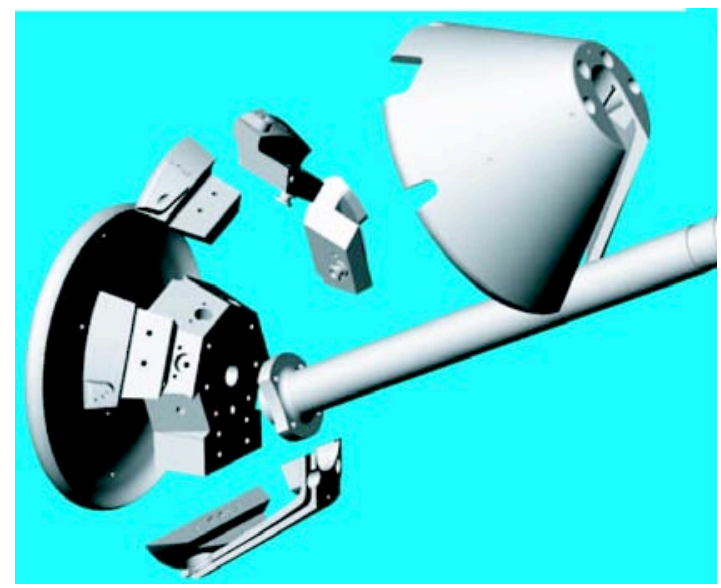

Figure 5. Expoded view for test model ACM-S24.

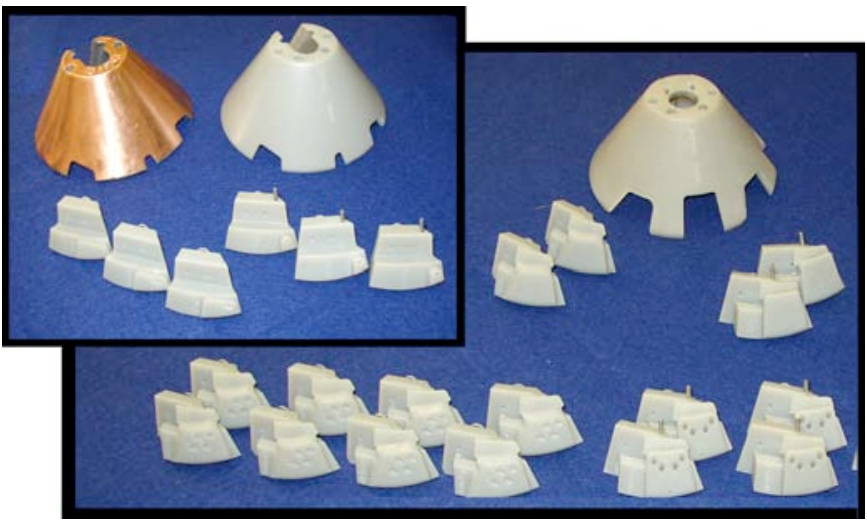

Figure 6. Aft-body pieces for the ACM (inset) and OCM models including various RCS jet configurations.

6

American Institute of Aeronautics and Astronautics 
capability, particularly for the small differences at the low pressures measured. Nozzle blocks are fabricated such that each is plumbed with 0.090 " diameter passages for the desired firing sequence. Different aft-body shells for the OCM and ACM model configurations and various nozzle blocks for both Bell and Cone nozzles and desired firing sequences are shown in Figure 6.

\section{Model Instrumentation}

There are five 0.040 " diameter pressure ports on the fore-body and eleven 0.060 " ports on the aft-body (nine on the aft-body shell plus one each on pitch and yaw nozzle blocks). Thin-skin thermocouples are also installed in the PSP aft body shells attached to the copper plating. To attach thermocouples, the SLA material is drilled out behind the desired surface locations and 30 gage type $\mathrm{K}$ thermocouple wire attached to the inside of the copper plating with silver epoxy. Discrete pressure and temperature measurements are used in the calibration of PSP data.

Other instrumentation in the model includes air pressure and temperature probes in the high-pressure manifold. Temperature and pressure sensing surfaces at the ends of threaded transducers are placed at the wall of a common cylindrical plenum $(0.3$ " x 0.25 " D), with equidistant passageways $(0.76$ " x 0.095 " diameter) to each of the nozzle blocks.

\section{Test Facility}

The Langley 31-Inch Mach 10 blow-down wind tunnel is used for this test. This tunnel uses dry air as the test gas and has a three-dimensional contoured nozzle and 31-inch square closed test section with a 14-inch inviscid core. Additional descriptions and specifications for this facility can be obtained from a report by Micol, 1998 .

\section{Flowfield Visualization Technique}

Nitric oxide seeded PLIF (NO-PLIF) is used for flowfield visualization. Details for this technique and additional results are presented by Inman, 2008. NO-PLIF uses an ultraviolet laser sheet to interrogate a slice in the flow containing seeded NO. NO-PLIF intensity is primarily a function of NO concentration and qualitatively shows the jet plume location, in which the NO is seeded. Several slices are taken in a series and reconstructed to visualize the flow in a volume around the jet plumes. The roll and yaw jets have highly 3-dimensional plume interaction flow fields. Once reconstructed, 3-dimensional volume data can be viewed and re-sectioned in a virtual computer environment.

For the ACM forward pitch jet a single planar PLIF image in the centerline symmetry plane also provides an opportunity for jet plume characterization in a single image capture. In the symmetry plane, individual jet plume shape, structure and expansion characteristics can be compared for each nozzle type (Danehy, 2008). Surface interactions for the pitch jet location, however, had been shown in earlier studies to be relatively benign, because of the large separated flow region on top of the vehicle. Surface measurements were not made for comparison in this test at that location.

\section{Surface Pressure and Temperature Mapping}

PSP and TSP is used to determine global pressure and temperature on the aft-body. Details for these techniques and additional results are presented by Watkins, 2008. The PSP painted surface is first electroplated with a thermally conductive copper ( 0.025 inches). In earlier experiments with PSP measurements, thermal gradients on the model surface were shown to interfere with the PSP pressure measurement, particularly for small differences at the low pressures measured for heating conditions in the Mach 10 tunnel. Comparisons of TSP heating measurements made with RCS jets on and off are used to determine heating augmentation attributed to interaction effects.

\section{Heat-Transfer Analysis}

Temperature data from the TSP coating was used to calculate the convective heat transfer coefficient. Calculations are based on a one-dimensional semi-infinite slab substrate with a constant heating coefficient and an initial condition of the substrate being at a uniform temperature. This is solved simply using an inverse polynomial fit to a well behaved implicit integral solution using the following equation:

$$
\frac{C_{h}}{C_{h F R}} \frac{\beta_{R E F}}{\beta}=z\left\{\Theta\left(T_{S}\right)\right\} \cdot \frac{\beta_{R E F}}{C_{h F R}} \cdot \frac{T_{S_{o}}}{h_{S_{o}}} \cdot\left(t-t_{R E F}\right)^{-1 / 2}
$$


where on the left hand side of the equation $\mathrm{C}_{\mathrm{h}}$ is the measured convective heat transfer coefficient based on enthalpy, $\mathrm{C}_{\mathrm{hFR}}$ is the reference convective heat transfer coefficient calculated using the method of Fay and Riddell, 1958, for a spherical nose radius of 6 inches. In the data analysis a reference value for substrate thermal product, $\beta_{\mathrm{REF}}$, is used. $\beta$ represents the exact value for the substrate thermal product (unknown at this time for the new SLA material) and heating results are presented as $\mathrm{C}_{\mathrm{h}} / \mathrm{C}_{\mathrm{hFR}}$ multiplied by $\beta_{\mathrm{REF}} / \beta$ to acknowledge a factor of error. Measurement of heating augmentation, $\mathrm{C}_{\mathrm{hA}} / \mathrm{C}_{\mathrm{hB}}$, is unaffected since $\beta_{\mathrm{REF}} / \beta$ is cancelled in the ratio.

On the right hand side of Equation 1, $z\left\{\Theta\left(T_{S}\right)\right\}$ is a function based on the normalized thermal parameter $\Theta$, where:

$$
\Theta\left(T_{S}\right) \equiv \frac{T_{S}(t)-T_{I N I T}}{T_{S_{o}}} \frac{h_{S_{o}}}{h_{a w}}
$$

and,

$$
z\{\Theta\}=\mathrm{a}_{0}+\mathrm{a}_{1} \Theta+\mathrm{a}_{2} \Theta^{2}+\mathrm{a}_{3} \Theta^{3}
$$

with the values for coefficients

$$
\begin{aligned}
& \mathrm{a}_{0}=-9.348 \times 10^{-6} \\
& \mathrm{a}_{1}=1.002 \\
& \mathrm{a}_{2}=1.374 \\
& \mathrm{a}_{3}=5.515
\end{aligned}
$$

$\mathrm{T}_{\mathrm{s}}$ is the measured surface temperature during the run. $\mathrm{T}_{\text {init }}$ is the initial measured surface temperature prior to injection. $T_{\text {so }}$ is the surface temperature used for calculating low temperature enthalpy $h_{\text {so }}$ (from which a constant specific heat is derived), and $\mathrm{h}_{\mathrm{aw}}$ is the adiabatic wall enthalpy, both of which are calculated from the GASPROPS code (Hollis, 1996) using measured tunnel flow conditions and gas properties. $t$ and $t_{\text {REF }}$ are the image time and initial reference time. Validation and further details on the derivation of this step heating approximation are contained in a CEV Aeroscience technical report (Buck, 2007).

\section{Experimental Results}

Surface pressure from PSP, heating coefficients from TSP temperatures and jet plume volume data from NOPLIF were obtained at approximate jet chamber pressures of $P_{j}=250$ psi and 500 psi for the ACM forward facing roll, yaw and OCM most windward yaw jet. Additional planar images were taken in the pitch jet symmetry plane with NO-PLIF. Flow conditions were set with stagnation pressures at $\mathrm{P}_{\mathrm{o}}=350 \mathrm{psi}$ and $1300 \mathrm{psi}$, at stagnation temperature of $\mathrm{T}_{\mathrm{o}}=1300^{\circ} \mathrm{F}$. These correspond to freestream Reynolds numbers of $\operatorname{Re}_{\infty, \mathrm{D}}=0.23 \times 10^{6}$ and $0.75 \times 10^{6}$ and jet pressure ratios of approximately $\mathrm{P}_{\mathrm{j}} / \mathrm{P}_{\mathrm{T}, 2}=60,120,200$ and 440 .

\section{Pitch-Jet Flowfield Images}

The location of NO-PLIF images relative to the test model for the ACM pitch jet symmetry plane is shown in Figure 7. A collection of single frame PLIF images are shown in Figure 8, providing clear comparisons between the two different nozzle plume shapes and interactions in the 2-dimensional plane. Comparisons of the plume shapes for the Bell and Cone nozzles $\left(\mathrm{A} / \mathrm{R}=13.4\right.$ and 22.5) are shown, both with the same nozzle throat diameter $\left(\mathrm{D}_{\mathrm{t}}=\right.$ 0.0275 ) and exit angle (10 degrees). The single frame images are also useful in showing flow mixing and turbulence, which is also shown to increase with increasing tunnel stagnation pressure.

Despite the large differences in area ratio, and nozzle shapes, a comparison of the upper and lower sets of images show that the plume shapes and mixing or turbulence are very similar at each of the different flow conditions; noflow (vacuum), and tunnel stagnation pressures $P_{o}=350$ psi and $P_{o}=1300$ psi for the jet pressure, $P_{j}=500$ psi case shown. Similar results were shown for $P_{j}=250$ psi (Danehy, 2008). Primary differences between the two nozzle plumes (Bell versus Cone) are shown to be internal to the jet plume. The external plume shapes are remarkably similar. The difference in internal plume structure is a spike appearing in the Bell plume in each of the cases but not for the Cone. Quasi-1D inviscid calculations show that the differences in the nozzle area ratios alone would cause 
condensation of the nitrogen gas in the Bell nozzle expansion but not in the Cone, with Mach number in the jet equal to 4.9 for the Bell $(A / R=22.5)$ versus 4.2 for the Cone $(A / R=13.4)$. Further discussion is given by Danehy, 2008 . The internal spike shown could also be a result of a focused non-uniformity which is typical for a poorly designed axi-symmetric, or a combination of both factors. Regardless, these results suggest that plume/flowfield interactions are similar, based on similarities in the outer plume shapes.

\section{Reconstructed 3-Dimensional ACM Roll and Yaw-Jet Flowfield Images}

Several PLIF images or slices were taken in a series to visualize the flow in a volume around the highly 3dimensional flow fields about the roll and yaw jets. Reconstruction of these volume slices for 3-dimensional viewing was done and combined with a virtual CAD model surface to be viewed readily in a computer virtual environment. Figure 9 are orthogonal and perspective views of this 3-dimensional database for the ACM forward facing roll jet. The reconstructed images are from a single test run, at tunnel conditions $\mathrm{P}_{\mathrm{o}}=1300$ psi and $\mathrm{T}_{\mathrm{o}}=1350$ ${ }^{\circ} \mathrm{F}$ and jet chamber pressure $\mathrm{P}_{\mathrm{j}}=250 \mathrm{psi}$. Additional reconstructions of measured jet plume volumes are presented by Inman, 2008. PSP pressure and TSP heating data are also projected onto the CAD model surface for that report.

\section{Surface Pressure Mappings}

Figures 10 and 11 are PSP pressure mappings with ACM forward roll and yaw jet interactions. These images compare well qualitatively with the following heating images. The resolution is somewhat low - but so are the pressures being measured ( 0.03 to $0.15 \mathrm{psi})$. False readings also indicate as negative values of pressure in these images. This is particularly apparent in Figure 10, in which the nozzle blocks were re-arranged to change firing configuration prior to the run. The exposed surface section of nozzle block for the roll jet was out of calibration with the rest of the aft-body being measured. Only one calibration was used over the entirety of each image and differences in coatings and exposure requires separate calibrations.

\section{Surface Heating and Heating Augmentation Mappings}

Figures 12 and 13 show the TSP heat-transfer mapping for the ACM forward roll and yaw jet surface interactions. The jet off images $\left(\mathrm{P}_{\mathrm{j}}=0\right)$ for all of these conditions (runs 29, 30, 38 and 37) should be similar (assuming laminar conditions) since they are all normalized to reference heating coefficients at each flow condition. Heat-transfer mappings compare well between flow conditions, $\mathrm{P}_{\mathrm{o}}=350$ psi and 1300 psi, but there are obvious differences between the roll jet (Fig. 12) and yaw jet (Fig. 13) model configurations for jets off. For one, the windward leading edge in the roll jet configuration shows higher heating, which was found to be caused by a misalignment between the aft-body shell and fore body resulting in a slight step. In the yaw jet configuration there is a line of higher heating towards the windward side further back on the aft body which is a result of a surface crack, which developed when refitting the aft-body to correct the windward step offset. These differences, however, are consistent between jets on and jets off for each of the cases and would later cancel in heating augmentation calculations (Figs. 15 and 16).

For the ACM forward roll jet the region of maximum heating concentration (area in the red) is shown to be affected more by flow conditions $\left(P_{o}=350\right.$ psi versus 1300 psi) than differences in jet chamber pressures $\left(P_{j}=250\right.$ psi versus $500 \mathrm{psi}$ ). The effect shown for differences in jet chamber pressures is actually opposite for the two sets of flow conditions. Heating levels for yaw jet interactions are mostly similar for each case, with interaction effects back slightly further on the aft-body for the high stagnation pressure.

Figure 14 shows a comparison of one of the yaw jet results $\left(P_{o}=350\right.$ psi, $P_{j}=500$ psi) with a simplified numerical model. This computational fluid dynamics (CFD) model incorporates laminar flow calculations with uniform jet outflow conditions. Both the experimental and numerical data show similar surface interaction heating patterns near the jet exit and similar heating levels relative to the rest of the aft-body. The experimental data, though, shows interaction heating extending much further back on the aft-body. Jet exit divergence angle was also shown numerically to significantly affect both the heat flux intensity and surface heating area on the aft-body, by as much as $25 \%$ increase in intensity and surface area for a 10 degree divergent jet versus parallel outflow. This sensitivity to jet exit divergence angle may factor significantly in planning future experiments.

Figures 15 and 16 show the heating augmentation in which the jet-on heating coefficient is normalized to jetoff condition. These data show significant heating augmentation to the surface, similar to the results shown by Jones and Hunt, 1965. Maximum heating augmentation values in Table 3 show tabulated results in very close agreement to the data shown in Table 1.

Heating augmentation for the OCM windward yaw jet are shown in Figure 17 for $\mathrm{P}_{\mathrm{j}}=250$ psi and 500 psi, for just the $\mathrm{P}_{\mathrm{o}}=350 \mathrm{psi}\left(\operatorname{Re}_{\infty, \mathrm{D}}=0.233 \times 10^{6}\right)$ case. The maximum heating augmentation values are tabulated in Table 4 for all of the flow conditions. The OCM yaw jet is swept back with the flow direction, not as perpendicular to the 
flow as the ACM configuration, and set back further on the vehicle. While the OCM maximum heating augmentation values are not lower than the ACM, the area affected by heating augmentation is much smaller as shown in these images.

\section{Conclusion}

A test was conducted in a Mach 10 hypersonic wind tunnel demonstrating and improving advanced measurement techniques that would aid in the rapid measurement and visualization of jet interaction effects for the Orion Crew Exploration Vehicle. An interchangeable capsule model was developed using a rapid prototyping capability in which the number of RCS jets, nozzle shapes, locations and piping (flow on or off) can be rapidly and inexpensively reconfigured.

Measurements included global surface imaging with pressure and temperature sensitive paints and threedimensional flow visualization with a scanning planar laser induced fluorescence technique. Measurements were focused primarily on the forward facing roll and yaw jet interactions on the capsule aft-body with an Apollo-like RCS jet configuration and were shown to compare well with earlier Apollo data, providing much higher resolution than previous methods which will be useful in comparing and validating CFD for future flight applications.

CFD modeling of the Apollo-like yaw jet configuration in the wind tunnel showed similar heating patterns, qualitatively, but also showed significant variation with jet exit divergence angle which may need to be explored further in future RCS jet plume interaction experiments.

Measurements on a preliminary Orion 4-cluster jet RCS configuration showed that measured heating augmentation levels are similar to yaw jet interactions on the Apollo-like configuration but with less area affected. These results along with the fabrication methods and advanced measurement techniques developed will be useful in the next phase of testing and evaluation of the updated Orion RCS configuration.

\section{Acknowledgements}

In an experiment of this complexity there were many involved in the process of designing, fabricating and executing the test. The authors would like to acknowledge in particular those who made significant contributions. Pete Vasquez provided exceptional help in the model design and fabrication. Anthony Robbins provided exceptional help, support and coordination in the design and fabrication of the high-pressure system. Kevin Hollingsworth was the principal facility engineer who was in charge of conducting the test and insuring the quality of the flow data. Don Oglesby was instrumental in the preparation and calibration of the PSP/TSP coating as well a Bradley Leighty and William Lipford in the preparation and test set up.

\section{References}

Buck, G. M., Powers, M. A., Griffith, M. S., Hopkins, J. W., Veneris, P. H., and Kuykendoll, K., "Fabrication of 0.0075-Scale Orbiter Phosphor Thermography Test Models for Shuttle RTF Aeroheating Studies," NASA TM 2006-214507, November 2006.

Buck, G. M., Watkins, A. N. and Danehy, P. M., "Aerothermodynamic Testing of RCS Jet Interactions on Apollo and Orion-type Crew Modules in the NASA Langley 31-Inch Mach 10 Air Tunnel: Test 37-CH," CEV Aeroscience Project Technical Brief, EG-CAP-07-97, 2007.

Danehy, P. M., Wilkes, J. A., Brauckmann, G., Alderfer, D. W., Jones, S. B., and Patry, D., "Visualization of a Capsule Entry Vehicle Reaction-Control System (RCS) Thruster” AIAA Paper 2006-1532, January 2006.

Danehy, P. M., Inman (Wilkes), J. A., Alderfer, D. W., and Buck, G. M., "Visualization of Flowfield Modification by RCS Jets on a Capsule Entry Vehicle" AIAA Paper 2008-1231, January 2008.

Fay, J. A., and Riddell, F. R., "Theory of Stagnation Point Heat Transfer in Dissociated Air," Journal of Aeronautical Sciences, Vol., 25, No., 2, 1958, pp. 73-85.

Hollis, B. R., "Real-Gas Flow Properties for NASA Langley Research Center Aerothermodynamic Facilities Complex Wind Tunnels," NASA Contractor Report 4755, September 1996.

Inman (Wilkes), J. A., Danehy, P. M., Alderfer, D. W., Buck, G. M., McCrea, A. and Schwartz, R. J., "PLIF Imaging of Capsule RCS Jets, Shear Layers, and Simulated Forebody Ablation" AIAA Paper 2008-248, January 2008. 
Jones, R. A. and Hunt, J. L., "Use of Temperature-Sensitive Coatings for Obtaining Quantitative Aerodynamic Heat-Transfer Data," AIAA Journal, Vol., 2, No., 7, July 1964.

Jones, R. A. and Hunt, J. L., "Effects of Cavities, Protuberances, and Reaction-Control Jets on Heat Transfer to the Apollo Command Module," NASA TM X-1063, March 1965.

Micol, J. R., "Langley Aerothermodynamics Facilities Complex: Enhancements and Testing Capabilities," AIAA Paper 98-0147, January 1998.

Scallion, W. I., "Space Shuttle Reaction Control System - Flow Field Interactions During Entry," Orbiter Experiments Aerothermodynamics Symposium, NASA Conf. Pub. 3248, part 1, ed. Throckmorton, D. A., 1995.

Spaid, F. W., and Cassel, L. A., “Aerodynamic Interference Induced by Reaction Controls," AGARD Report AG173, 1973.

Watkins, A. N., Buck, G. M., Leighty, B. D., and Lipford, W. E., "Using Pressure- and Temperature-Sensitive Paint for Global Surface Pressure and Temperature Measurements on the Aft-Body of a Capsule Reentry Vehicle" AIAA Paper 2008-1230, January 2008.

Table 3. List of Current Test Conditions and Maximum Heating Augmentation Values for ACM RCS Jet Interference Heating

\begin{tabular}{ccccccc}
\hline \hline RCS Jet & $\boldsymbol{D}_{\boldsymbol{t}}$ (in.) & $\boldsymbol{R e}_{\infty, \boldsymbol{D}} \boldsymbol{x} \mathbf{1 0} \mathbf{0}^{\mathbf{6}}$ & $\boldsymbol{P}_{\boldsymbol{j}} / \boldsymbol{P}_{\boldsymbol{T}, \boldsymbol{2}}$ & $\boldsymbol{P}_{\boldsymbol{j}},(\mathbf{p s i})$ & $\boldsymbol{C}_{\boldsymbol{h} \boldsymbol{A}} / \boldsymbol{C}_{\boldsymbol{h} \boldsymbol{B}}$ & $\mathbf{R u n}$ \\
\hline forward roll & 0.028 & 0.738 & 63 & 254 & 5 & 32 \\
forward roll & 0.028 & 0.754 & 122 & 495 & 5 & 34 \\
forward roll & 0.028 & 0.233 & 224 & 253 & $10+$ & 31 \\
forward roll & 0.028 & 0.233 & 425 & 481 & $10+$ & 33 \\
yaw & 0.028 & 0.746 & 63 & 254 & 5 & 41 \\
yaw & 0.028 & 0.763 & 125 & 507 & 5 & 36 \\
yaw & 0.028 & 0.433 & 200 & 497 & 5 & 42 \\
yaw & 0.028 & 0.233 & 448 & 508 & 5 & 39 \\
\hline \hline
\end{tabular}

Table 4. List of Current Test Conditions and Maximum Heating Augmentation Values for OCM RCS Jet Interference Heating

\begin{tabular}{|c|c|c|c|c|c|c|}
\hline RCS Jet & $D_{t}$ (in.) & $R e_{\infty, D} x 10^{6}$ & $\boldsymbol{P}_{j} / \boldsymbol{P}_{T, 2}$ & $P_{j},($ psi) & $C_{h A} / C_{h B}$ & Run \\
\hline windward yaw & 0.028 & 0.746 & 119 & 478 & 4 & 50 \\
\hline " " & 0.028 & 0.229 & 56 & 236 & 5 & 51 \\
\hline$"$ & 0.028 & 0.233 & 447 & 507 & 6 & 47 \\
\hline
\end{tabular}
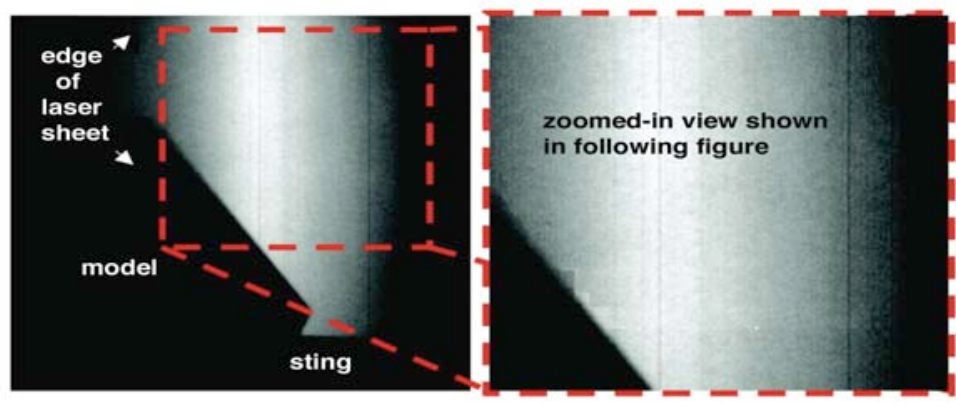

Figure 7. Location for Pitch Jet Planar PLIF images shown in following figure. 


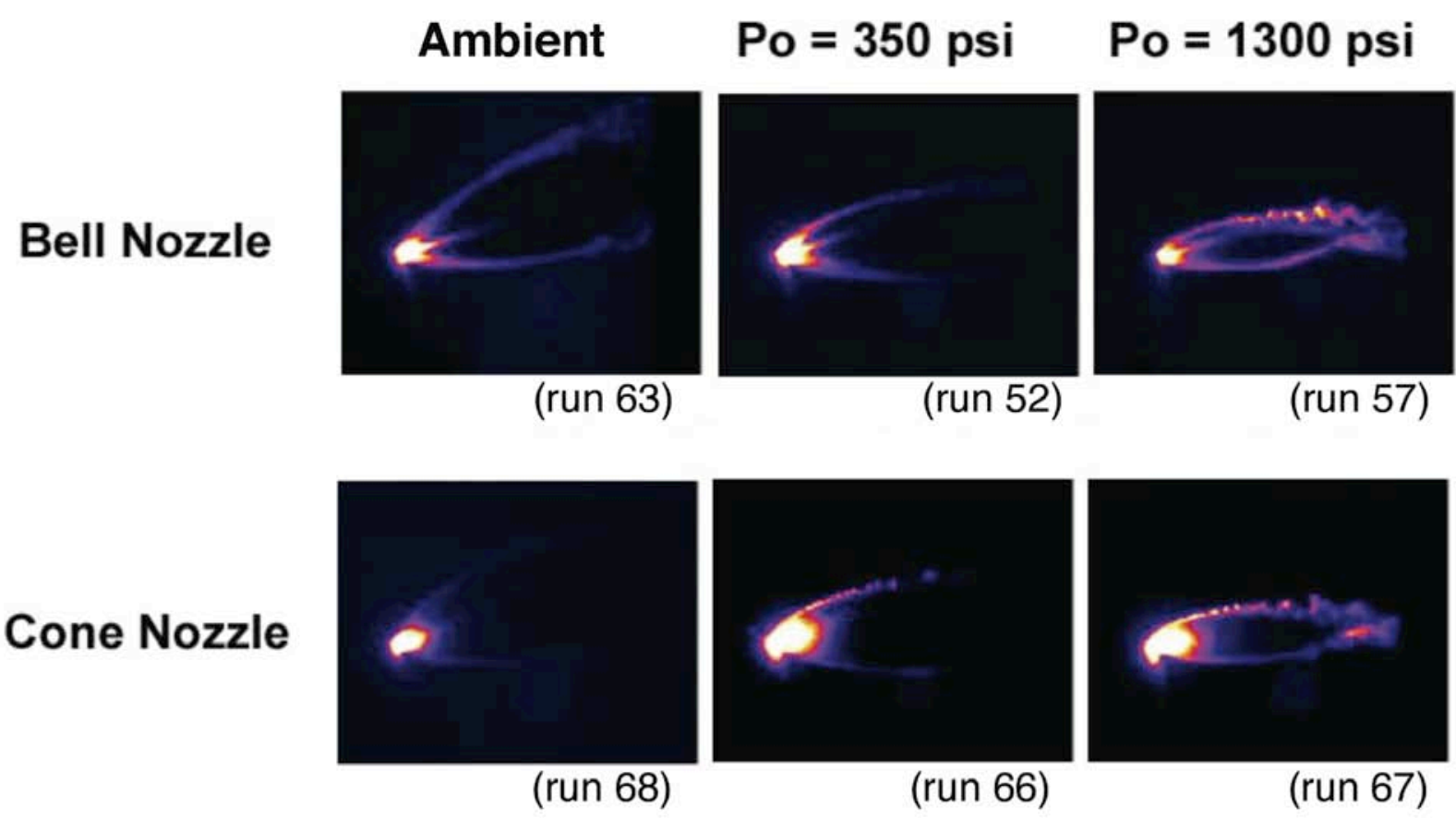

Figure 8. Planar PLIF Flow Visualization of ACM Pitch Jet, Single Frame, Pj 500 psi.
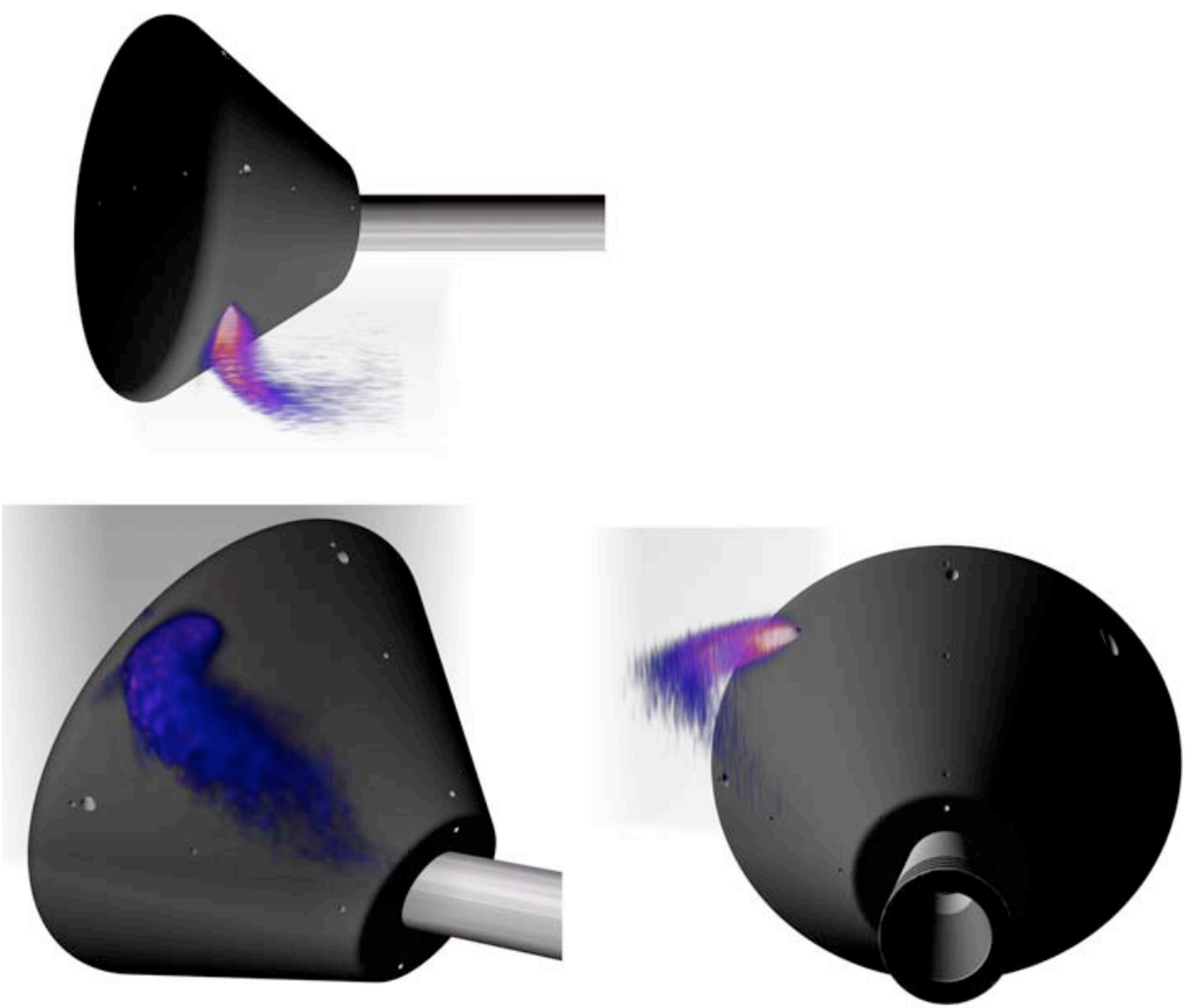

Figure 9. Reconstructed 3-Dimensional PLIF jet plume, $A C M$ roll-jet, $P j=250 \mathrm{psi}, \mathrm{Po}=1300 \mathrm{psi}$. 


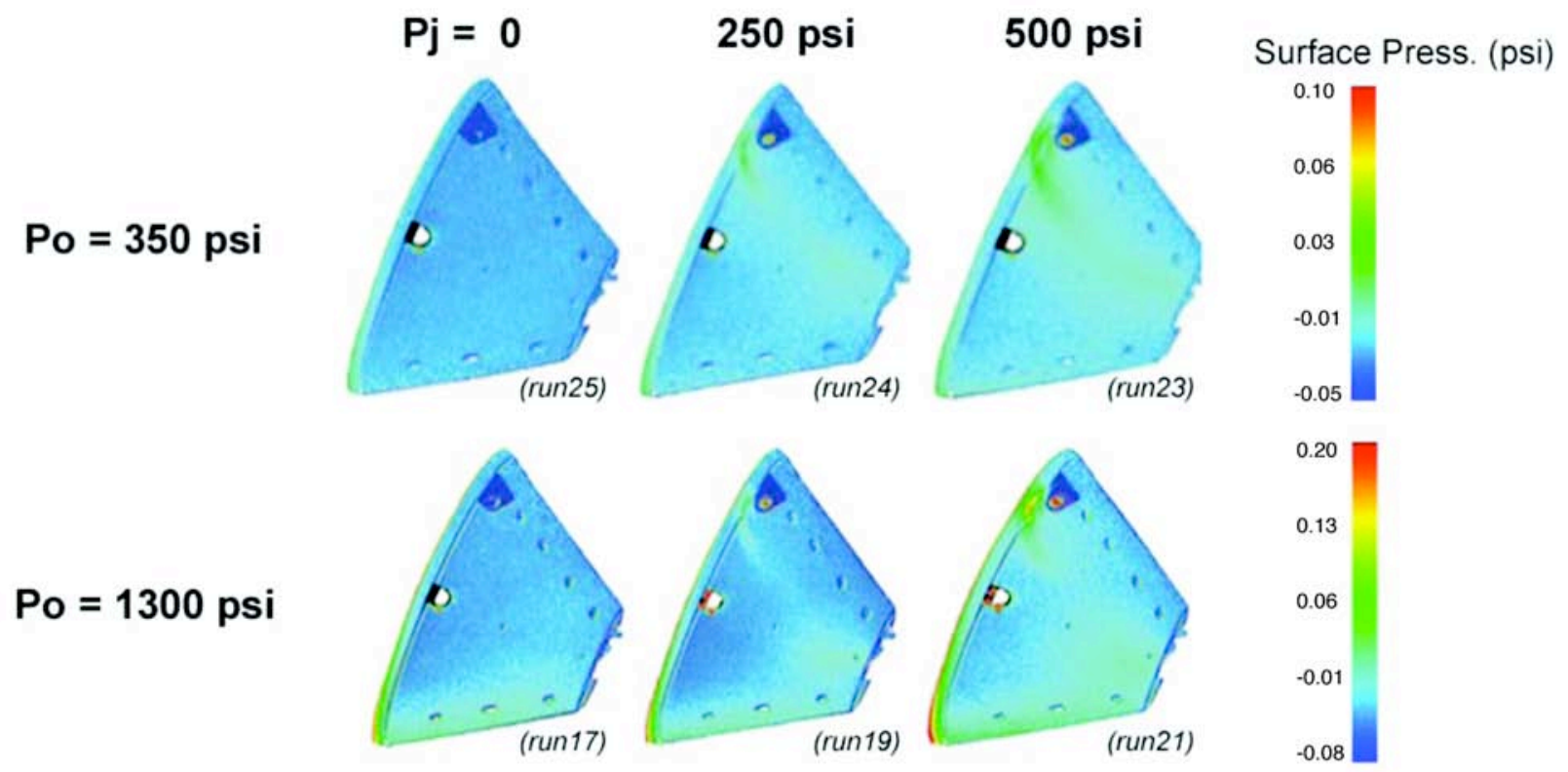

Figure 10. PSP Pressure Mapping of ACM Roll Jet Surface Interactions.

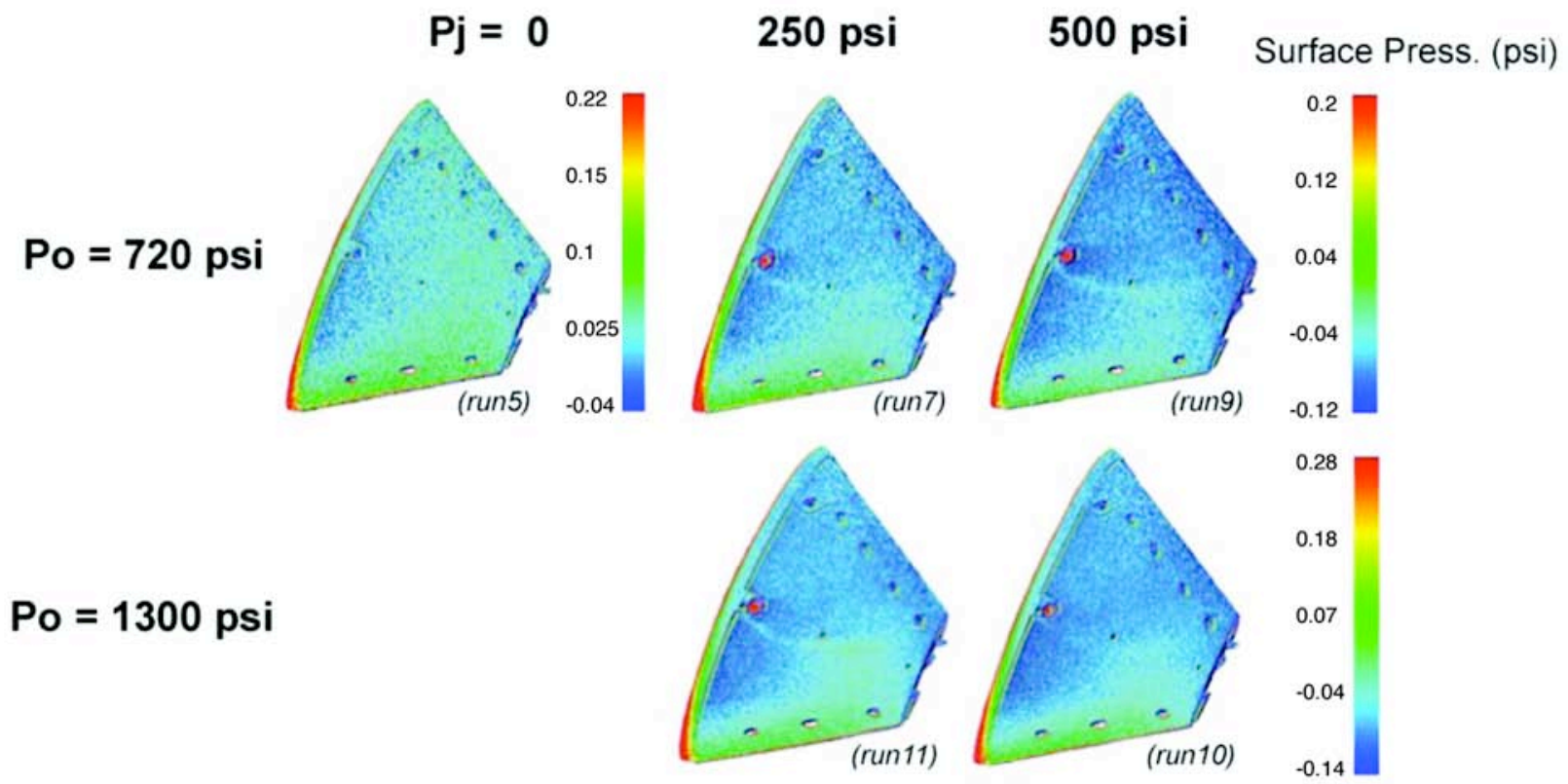

Figure 11. PSP Pressure Mapping of ACM Yaw Jet Surface Interactions. 


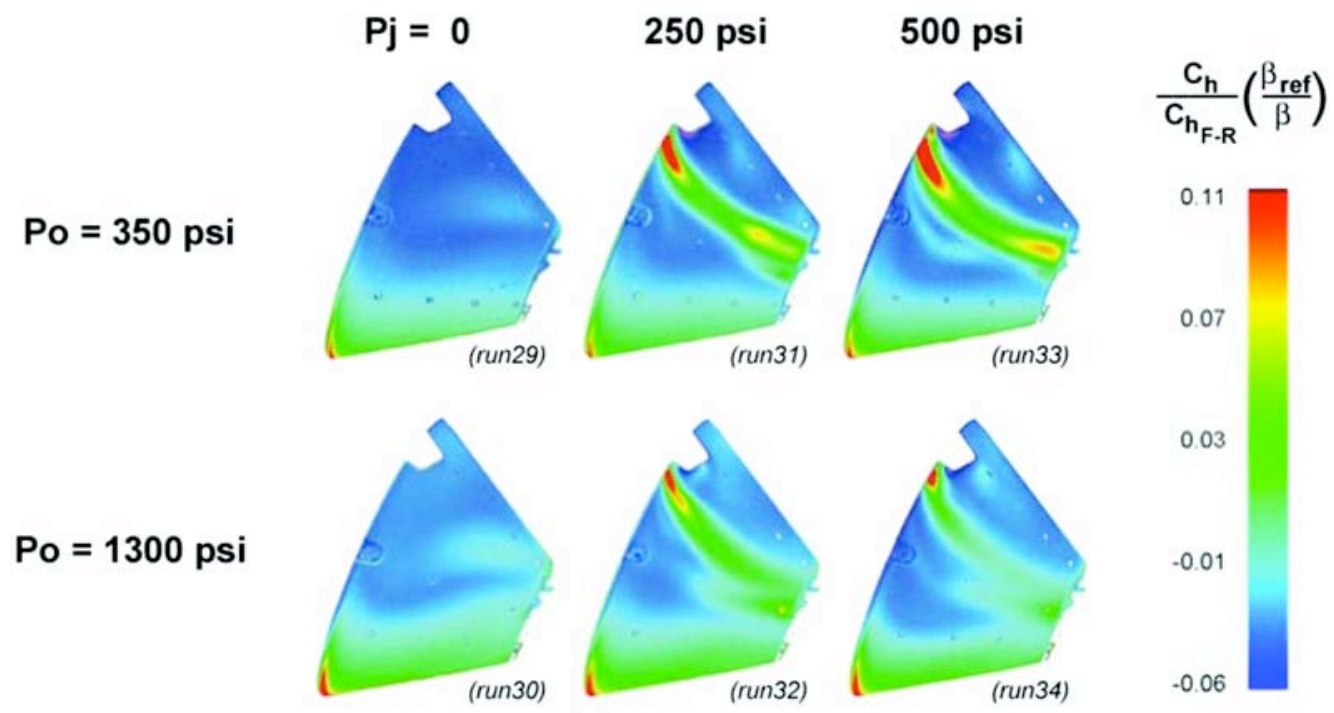

Figure 12. TSP Heat-Transfer Mapping of ACM Forward Roll Jet Surface Interactions.

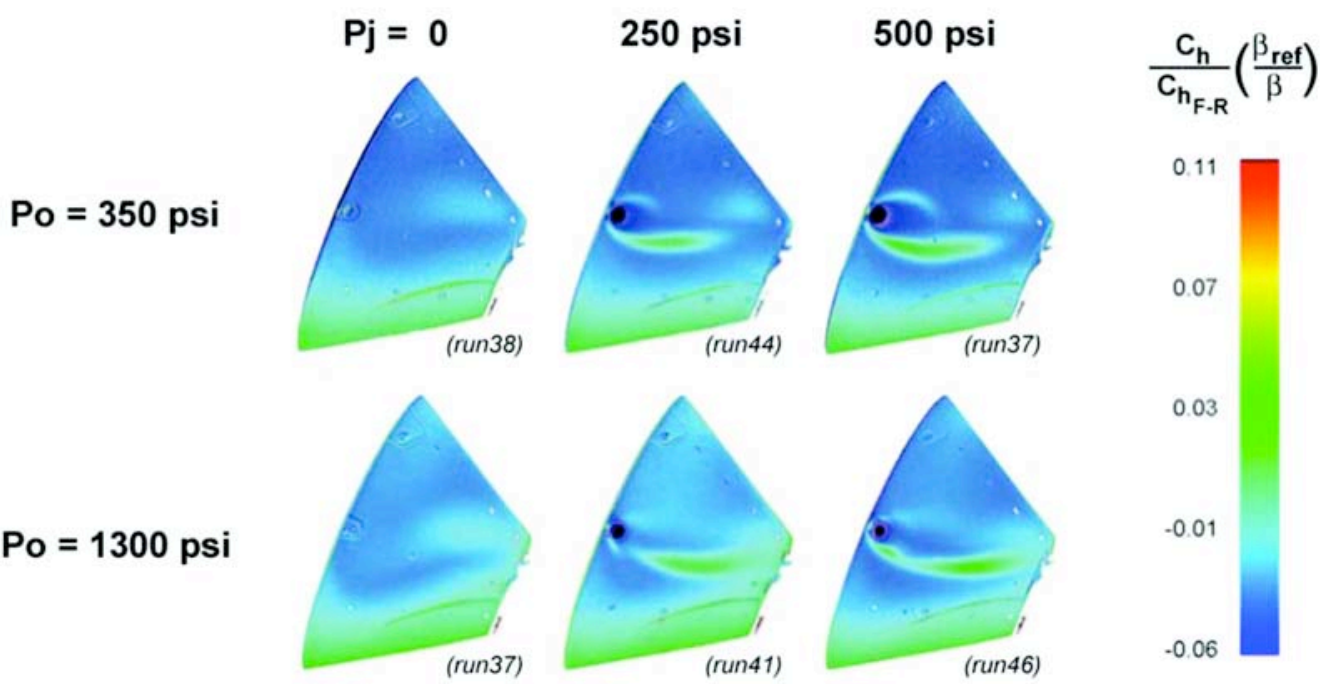

Figure 13. TSP Heat-Transfer Mapping of ACM Yaw Jet Surface Interactions.

$\dot{\mathrm{q}}_{\mathrm{s}}\left(\mathrm{W} / \mathrm{cm}^{2}\right)$

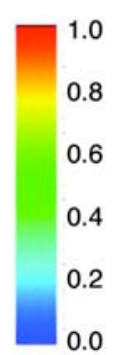

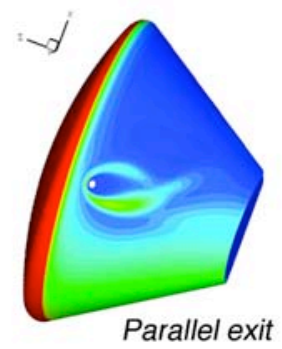

Computation

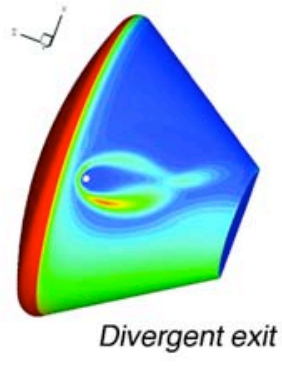

Divergent exit

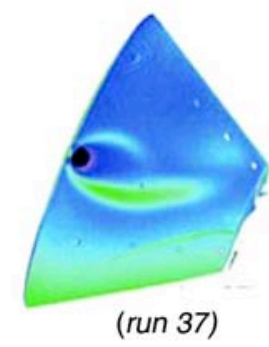

Experiment
$\frac{C_{h}}{C_{h_{F} \cdot R}}\left(\frac{\beta_{\text {ref }}}{\beta}\right)$

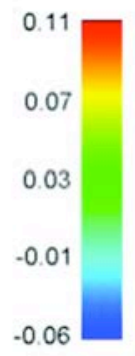

.0 .06

Figure 14. Comparison to Computation for ACM Yaw Jet Interactions, $P o=350 \mathrm{psi}, \mathrm{Pj}=500 \mathrm{psi}$. 


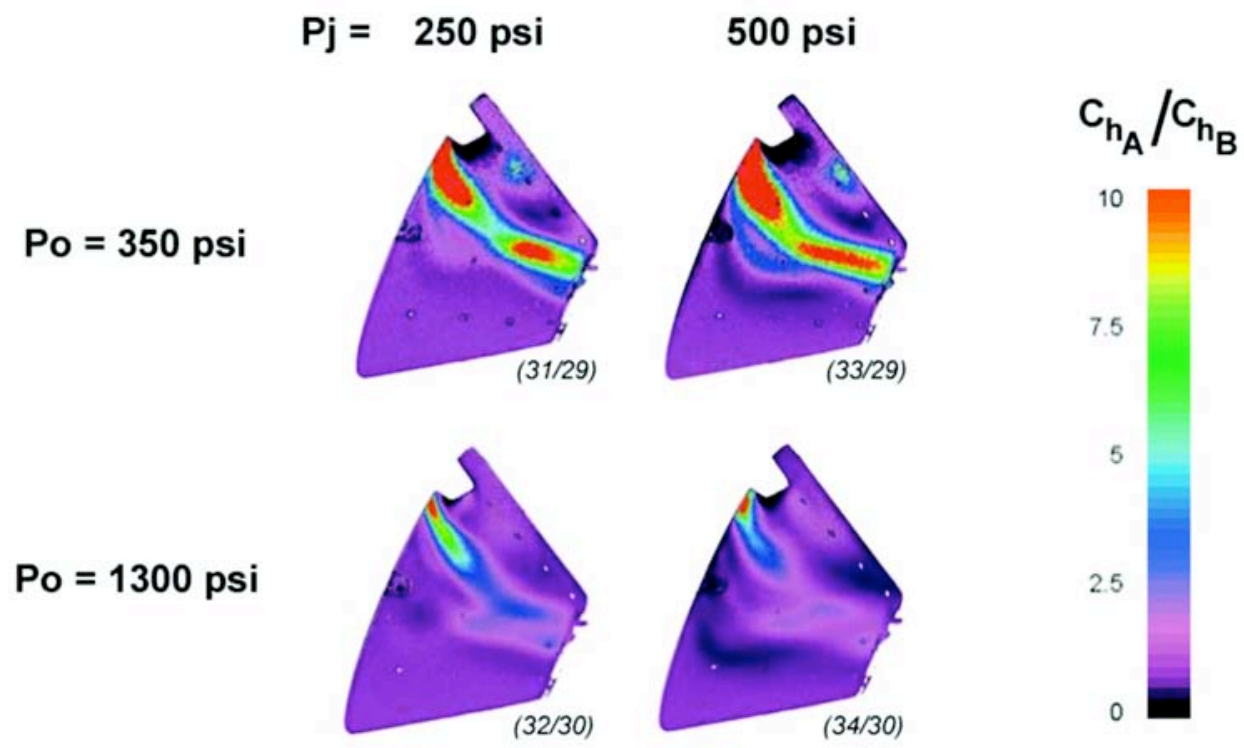

Figure 15. Heating Augmentation Mapping of ACM Forward Roll Jet Surface Interactions.

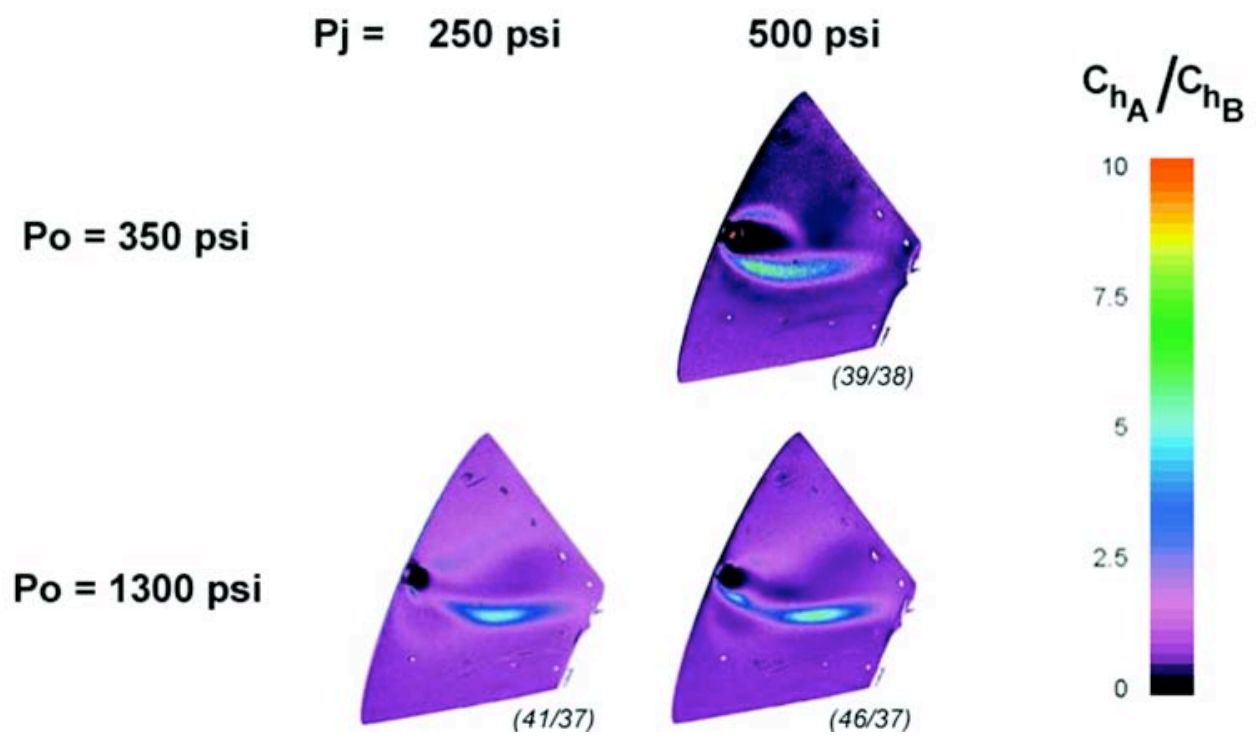

Figure 16. Heating Augmentation Mapping of ACM Yaw Jet Surface Interactions.

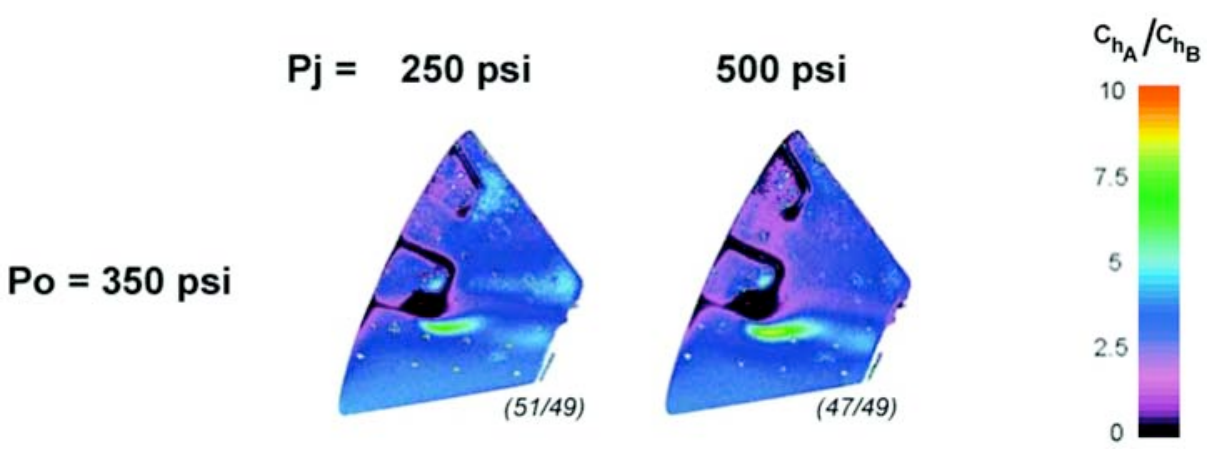

Figure 17. Heating Augmentation Mapping of OCM Windward Yaw Jet Surface Interactions. 